Article

\title{
Fractional-Order Logistic Differential Equation with Mittag-Leffler-Type Kernel
}

\author{
Iván Area ${ }^{1, *,+}(\mathbb{D})$ and Juan J. Nieto ${ }^{2,+}$ (D) \\ 1 CITMAga, Universidade de Vigo, Departamento de Matemática Aplicada II, E.E. Aeronáutica e do Espazo, \\ Campus As Lagoas-Ourense, 32004 Ourense, Spain \\ 2 CITMAga, Instituto de Matemáticas, Universidade de Santiago de Compostela, \\ 15782 Santiago de Compostela, Spain; juanjose.nieto.roig@usc.es \\ * Correspondence: area@uvigo.gal \\ + Both authors contributed equally to this work.
}

check for updates

Citation: Area, I.; Nieto, J.J. Fractional-Order Logistic Differential Equation with Mittag-Leffler Type Kernel. Fractal Fract. 2021, 5, 273. https://doi.org/10.3390/ fractalfract5040273

Academic Editor: Hari Mohan Srivastava

Received: 10 November 2021 Accepted: 9 December 2021 Published: 14 December 2021

Publisher's Note: MDPI stays neutral with regard to jurisdictional claims in published maps and institutional affiliations.

Copyright: (c) 2021 by the authors. Licensee MDPI, Basel, Switzerland. This article is an open access article distributed under the terms and conditions of the Creative Commons Attribution (CC BY) license (https:// creativecommons.org/licenses/by/ $4.0 /)$.
Abstract: In this paper, we consider the Prabhakar fractional logistic differential equation. By using appropriate limit relations, we recover some other logistic differential equations, giving representations of each solution in terms of a formal power series. Some numerical approximations are implemented by using truncated series.

Keywords: logistic differential equation; fractional calculus; Liouville-Caputo fractional derivative; Atangana-Baleanu derivative; Caputo-Fabrizio derivative; Prabhakar derivative; Prabhakar integral

MSC: Primary 34A08; secondary 65Q30

\section{Introduction}

Let us consider the classical logistic differential equation

$$
x^{\prime}(t)=x(t)(1-x(t))
$$

which can be explicitly solved. The constant solutions are $x(t)=0$ and $x(t)=1$. If another initial condition $x(0)=x_{0}$ is imposed, the solution is given by

$$
x(t)=\frac{x_{0}}{x_{0}+\left(1-x_{0}\right) \exp (-t)} .
$$

The solution can be also obtained in terms of formal power series. Let

$$
x(t)=\sum_{n=0}^{\infty} a_{n} t^{n}
$$

Then,

$$
x^{\prime}(t)=\sum_{n=1}^{\infty} n a_{n} t^{n-1}=\sum_{n=0}^{\infty}(n+1) a_{n+1} t^{n}
$$

and

$$
x^{2}(t)=\sum_{n=0}^{\infty}\left(\sum_{j=0}^{n} a_{j} a_{n-j}\right) t^{n}
$$

By substituting into (1), we obtain the following recurrence relation for the coefficients

$$
a_{n+1}=\frac{1}{n+1}\left[a_{n}-\sum_{j=0}^{n} a_{j} a_{n-j}\right], \quad n \geq 1, \quad a_{0}=x(0),
$$


which provide a solution in a neighbourhood of $t=0$ as described in [1].

It is possible to obtain the same recurrence relation if we further apply the Laplace transform to (1). Let $x(t)$ be given in (2), so that (3) holds true. Let $\mathcal{L}$ denote the Laplace transform, and as usual, we shall denote $F(s)$ the Laplace transform of a function $f(t)$. Then,

$$
\mathcal{L}[x(t)(1-x(t))]=\mathcal{L}\left[x(t)-x^{2}(t)\right]=\sum_{n=0}^{\infty}\left(a_{n}-b_{n}\right) \frac{n !}{s^{n+1}}, \quad b_{n}=\sum_{j=0}^{n} a_{j} a_{n-j} .
$$

Moreover,

$$
\mathcal{L}\left[x^{\prime}(t)\right]=s X(s)=\sum_{n=0}^{\infty} a_{n+1}(n+1) \frac{n !}{s^{n+1}}
$$

Thus,

$$
\sum_{n=0}^{\infty} a_{n+1}(n+1) \frac{n !}{s^{n+1}}=\sum_{n=0}^{\infty}\left(a_{n}-b_{n}\right) \frac{n !}{s^{n+1}}
$$

which implies again the recurrence relation (4) for the coefficients of the power series expansion of the solution. We need to impose that $a_{0}=x(0)$ to be able to start the latter recurrence relation.

We have included in Figure 1 some plots by using Mathematica [2] of the logistic function, solution to (1) with $x(0)=1 / 2$, and approximations of the function by the corresponding Taylor polynomials.

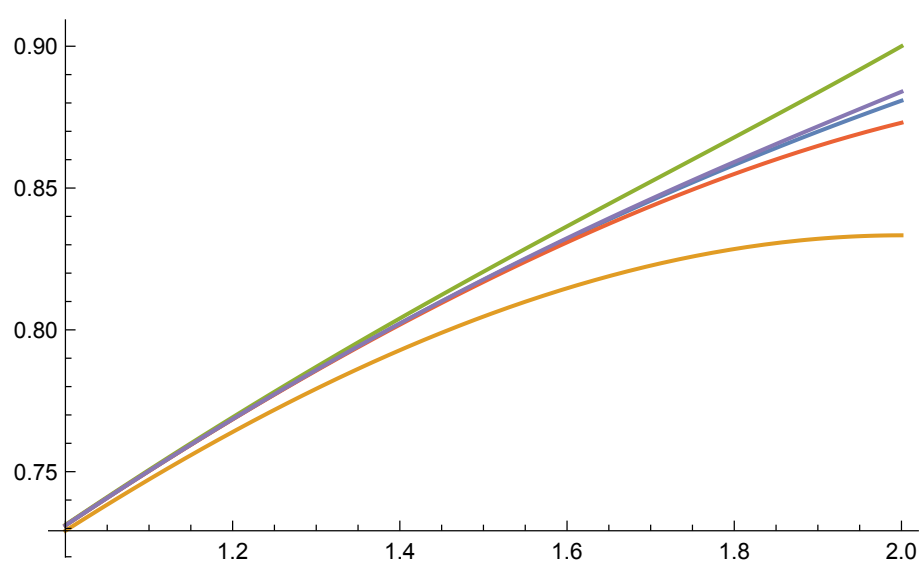

Figure 1. Logistic function solution to Equation (1) with $x(0)=1 / 2$, in blue, as well approximations of the function by the corresponding Taylor polynomials in [1,2]: $n=3$ in orange color, $n=5$ in green color, $n=7$ in red color, and $n=9$ in grey color.

This very classical logistic differential equation has been deeply studied due to its applications in different fields [3]. Recently, it has been used to study the evolution of the COVID-19 pandemic [4,5]. By considering fractional derivatives, the fractional analogue has been analyzed in several works mainly by considering the Liouville-Caputo fractional derivative [1,6-11] (see also [12-14]). The classical logistic ordinary differential equation has been recently studied from the view of fractional calculus and solved in some particular cases $[1,8,10]$. In this work, we consider the fractional logistic differential equation by using the Prabhakar fractional calculus [15-17].

The main aim of this work is to present the Prabhakar fractional logistic differential equation and, by appropriate limit transitions, recover several logistic differential equations (Liouville-Caputo, Atangana-Baleanu, and Caputo-Fabrizio), providing in each case a representation of the expansion of the solution in formal power series.

For the fractional Prabhakar logistic differential equation, we know the solutions for the Liouville-Caputo fractional derivative [7] (in terms of power series) and for the Caputo-Fabrizio derivative [18] (in implicit form). We emphasize that in this work we present the solution in terms of a power series expansion, as compared with the previous 
work [18], in which the solution is given in implicit form. It is also important to notice the fact that much more general fractional-calculus operators are available in the literature survey [19].

The structure of this work is the following: in Section 2 basic definitions, notations and results are presented. In Section 3 the Prabhakar fractional logistic equation is presented. For specific values of the parameters we recover the Liouville-Caputo, Atangana-Baleanu, and Caputo-Fabrizio logistic differential equations. For each of these cases, the solution is computed in terms of a formal power series. Some numerical experiments are also presented.

\section{Basic Definition and Notations}

Let $\alpha \in(0,1)$ and $\sigma \in L^{1}(0,1)$. The (Riemann-Liouville) fractional integral is defined by

$$
I^{\alpha} \sigma(t)=\frac{1}{\Gamma(\alpha)} \int_{0}^{t}(t-s)^{\alpha-1} \sigma(s) d s, \quad t \in(0,1)
$$

where $\Gamma(z)$ denotes the Euler gamma function [20].

For $z \in \mathbb{C}, \alpha, \beta, \gamma \in \mathbb{C}$ with $\Re(\alpha)>0$, the three-parameter Mittag-Leffler function, introduced by Prabhakar in 1971 [16], is defined by

$$
E_{\alpha, \beta}^{\gamma}(z)=\sum_{n=0}^{\infty} \frac{(\gamma)_{n}}{\Gamma(n \alpha+\beta)} \frac{z^{n}}{n !},
$$

which generalizes both the Mittag-Leffler function $(\gamma=1)$ as well as the classical exponential function $(\alpha=\beta=\gamma=1)$. Additionally, $E_{\alpha, \beta}^{0}(z)=1 / \Gamma(\beta)$. We would like to emphasize

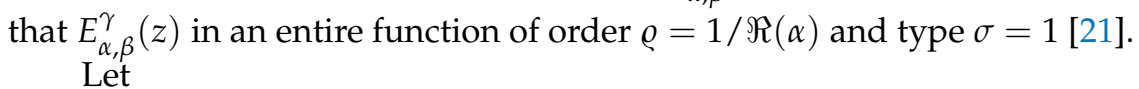

$$
e_{\alpha, \beta}^{\gamma}(\lambda ; t)=t^{\beta-1} E_{\alpha, \beta}^{\gamma}\left(\lambda t^{\alpha}\right),
$$

be the Prabhakar kernel. In particular,

$$
e_{1,1}^{1}(\lambda ; t)=\exp (\lambda t) ; \quad e_{\alpha, \beta}^{0}(\lambda ; t)=\frac{t^{\beta-1}}{\Gamma(\beta)}
$$

Additionally,

$$
e_{\alpha, \beta}^{\gamma}(0 ; t)=\frac{t^{\beta-1}}{\Gamma(\beta)} .
$$

The Prabhakar fractional integral with base point 0 is defined by

$$
\mathbb{P}_{\alpha, \beta, \lambda}^{\gamma} \sigma(t)=\int_{0}^{t} e_{\alpha, \beta}^{\gamma}(\lambda ; t-s) \sigma(s) d s
$$

for $\sigma \in L^{1}(0,1)$. For $\sigma \in L^{1}(0,1)$,

$$
\mathbb{P}_{\alpha, \beta, \lambda}^{\gamma} \sigma(t)=\sum_{n=0}^{\infty} \frac{(\gamma)_{n} \lambda^{n}}{n !} I^{\alpha n+\beta} \sigma(t)
$$

Thus, the Prabhakar fractional integral $\mathbb{P}_{\alpha, \beta, \lambda}^{\gamma}$ is linear and bounded from $L^{p}(0,1)$ into $L^{p}(0,1)$ for any $1 \leq p \leq \infty$.

Recall that taking $\lambda=-1$

$$
e_{\alpha, \beta}^{\gamma}(-1, t)=t^{\beta-1} E_{\alpha, \beta}^{\gamma}\left(-t^{\alpha}\right)
$$

is completely monotone if $0<\alpha \gamma \leq b \leq 1$ [22]. For example, $e_{1,1}^{1}(-1, t)=\exp (-t)$.

Moreover, Ref. [15]

$$
\mathbb{P}_{\alpha, \beta, \lambda}^{\gamma} e_{\alpha, \mu}^{\omega}(\lambda ; t)=e_{\alpha, \beta+\mu}^{\gamma+\omega}(\lambda ; t) .
$$


Let $\sigma \in L^{1}(0,1), \Re(\alpha)>0, \Re(\beta)>0$. The Prabhakar fractional derivative in the Riemann-Liouville sense is defined by

$$
\mathbb{D}_{\alpha, \beta, \lambda}^{\gamma} \sigma(t)=\frac{d}{d t} \mathbb{P}_{\alpha, 1-\beta, \lambda}^{-\gamma} \sigma(t)
$$

In doing so, it is required some regularity for $\sigma$, for example, that $\sigma \star e_{\alpha, 1-\beta, \lambda}^{-\gamma} \in W^{1,1}(0,1)$, where for $\Omega \subset \mathbf{R}^{n}$ the Sobolev space $W^{m, p}(\Omega)$ is defined by

$$
W^{m, p}(\Omega)=\left\{f \in L^{p}(\Omega)\left|D^{\alpha} \in L^{p}(\Omega), \forall \alpha \in \mathbf{N}^{n}:\right| \alpha \mid \leq m\right\} .
$$

The Prabhakar fractional derivative in the Liouville-Caputo sense is

$$
\mathbb{D}_{\alpha, \beta, \lambda}^{\gamma} \sigma(t)=\mathbb{P}_{\alpha, 1-\beta, \lambda}^{-\gamma} \sigma^{\prime}(t)
$$

We note that

$$
\mathbb{P}_{\alpha, \beta, 1}^{0} \sigma(t)=\int_{0}^{t} e_{\alpha, \beta}^{0}(\lambda, t-s) \sigma(s) d s=\frac{1}{\Gamma(\beta)} \int_{0}^{t}(t-s)^{\beta-1} \sigma(s) d s=I^{\beta} \sigma(t) .
$$

Additionally,

$$
\mathbb{P}_{\alpha, \beta, 0}^{\gamma} \sigma(t)=\int_{0}^{t} e_{\alpha, \beta}^{\gamma}(0, t-s) \sigma(s) d s=\frac{1}{\Gamma(\beta)} \int_{0}^{t}(t-s)^{\beta-1} \sigma(s) d s=I^{\beta} \sigma(t) .
$$

In both cases, the classical Riemann-Liouville integral of order $\beta>0$ is a particular case of the Prabhakar operator.

The Laplace transform of the Prabhakar fractional derivative in the Liouville-Caputo sense is [15] (page 27, Section 5.1, Equation (5.13))

$$
\mathcal{L}\left[\mathbb{D}_{\alpha, \beta, \lambda}^{\gamma} x(t)\right](s)=s^{\beta-\alpha \gamma}\left(s^{\alpha}-\lambda\right)^{\gamma}\left\{\mathcal{L}[x(t)](s)-\sum_{k=0}^{m-1} s^{-k-1} f^{(k)}(0+)\right\},
$$

where $m$ denotes the integer part of $\beta$. In particular, if $m=0$ or $m=1$, we have

$$
\mathcal{L}\left[\mathbb{D}_{\alpha, \beta, \lambda}^{\gamma} t^{n}\right](s)=s^{\beta-\alpha \gamma}\left(s^{\alpha}-\lambda\right)^{\gamma} \frac{\Gamma(n+1)}{s^{n+1}} .
$$

Let us consider the Liouville-Caputo fractional derivative [23] for an absolutely continuous function $f:[0, T] \mapsto \mathbb{R}$

$$
\mathrm{C}_{\mathbb{D}^{\alpha}} f(t)=\frac{1}{\Gamma(1-\alpha)} \int_{0}^{t}(t-s)^{-\alpha} f^{\prime}(s) d s, \quad t \in[0, T] .
$$

We have that

$$
\mathcal{L}\left[{ }^{C} \mathbb{D}^{\alpha} t^{n}\right](s)=\frac{\Gamma(n+1)}{s^{n-\alpha+1}}, \quad \alpha>0
$$

Since

$$
\mathcal{L}\left[\mathbb{D}_{\alpha, \beta, 0}^{\gamma} t^{n}\right](s)=\mathcal{L}\left[\mathbb{D}_{\alpha, \beta, t}^{0} t^{n}\right](s)=\frac{\Gamma(n+1)}{s^{n-\beta+1}}=\mathcal{L}\left[{ }^{C} \mathbb{D}^{\beta} t^{n}\right](s) .
$$

the Liouville-Caputo fractional derivative is a particular case of the Prabhakar fractional derivative.

The Atangana-Baleanu operator in the sense of Caputo for $u \in \operatorname{AC}(0,1)=W^{1,1}(0,1)$ is defined by [24]

$$
\mathrm{AB}_{\mathbb{D}^{\alpha}} u(t)=\frac{1}{1-\alpha} \int_{0}^{t} E_{\alpha}\left(-\frac{\alpha}{1-\alpha}(t-s)^{\alpha}\right) u^{\prime}(s) d s .
$$


It yields

$$
\mathcal{L}\left[{ }^{\mathrm{AB}} \mathbb{D}^{\alpha} t^{n}\right](s)=\frac{B(\alpha)}{1-\alpha} \frac{1}{s^{\alpha}+\frac{\alpha}{1-\alpha}} \frac{\Gamma(n+1)}{s^{n-\alpha+1}} .
$$

where $B(\alpha)$ is a normalizing function satisfying $B(0)=B(1)=1$. Let $\beta=0, \gamma=-1$, $\lambda=\alpha /(\alpha-1)$, so that

$$
\mathcal{L}\left[\mathbb{D}_{\alpha, 0, \alpha /(\alpha-1)}^{-1} t^{n}\right](s)=\frac{\Gamma(n+1) s^{\alpha-n-1}}{s^{\alpha}+\frac{\alpha}{1-\alpha}}=\frac{1-\alpha}{B(\alpha)} \mathcal{L}\left[{ }^{\mathrm{AB}} \mathbb{D}^{\alpha} t^{n}\right](s) .
$$

As a consequence, the Atangana-Baleanu derivative is a particular case of the Prabhakar fractional derivative.

For $u \in \mathrm{AC}(0,1)=W^{1,1}(0,1)$, the Caputo-Fabrizio fractional derivative is defined by [25] (see also [26])

$$
\mathrm{CF}_{\mathbb{D}^{\alpha}} u(t)=\frac{1}{1-\alpha} \int_{0}^{t} \exp \left(-\frac{\alpha}{1-\alpha}(t-s)\right) u^{\prime}(s) d s .
$$

We have that

$$
\mathcal{L}\left[{ }^{\mathrm{CF}} \mathbb{D}^{\alpha} t^{n}\right](s)=\frac{\Gamma(n+1)}{\alpha+(1-\alpha) s} \frac{1}{s^{n}} .
$$

Let $\alpha=1, \beta=0, \gamma=-1, \lambda=\alpha /(\alpha-1)$, so that

$$
\mathcal{L}\left[\mathbb{D}_{1,0, \alpha /(\alpha-1)}^{1} t^{n}\right](s)=\frac{s^{-n} \Gamma(n+1)}{s-\frac{\alpha}{\alpha-1}}=(1-\alpha) \mathcal{L}\left[{ }^{\mathrm{CF}} \mathbb{D}^{\alpha} t^{n}\right](s),
$$

revealing that the Caputo-Fabrizio derivative [25] is also a particular case of the Prabhakar fractional derivative.

\section{Prabhakar Fractional Logistic Equation and Its Limiting Cases}

Let $\mathbb{D}_{\alpha, \beta, \lambda}^{\gamma} x(t)$ be the Prabhakar fractional derivative of a given function $x(t)$. Let us now consider the Prabhakar fractional logistic differential equation

$$
\Lambda(\alpha, \beta, \gamma, \lambda) \mathbb{D}_{\alpha, \beta, \lambda}^{\gamma} x(t)=x(t)(1-x(t)),
$$

where the constant $\Lambda(\alpha, \beta, \gamma, \lambda)$ is defined by

$$
\Lambda(\alpha, \beta, \gamma, \lambda)= \begin{cases}1-\frac{\lambda}{\lambda-1}, & \alpha=1 \\ \left(\frac{B(\alpha)}{1-\alpha}\right)^{\frac{(1-\alpha) \gamma \lambda}{\alpha}}, & \alpha \neq 1 .\end{cases}
$$

Let

$$
x(t)=\sum_{n=0}^{\infty} a_{n} t^{n \tau}
$$

so that

$$
x(t)(1-x(t))=\sum_{n=0}^{\infty}\left(a_{n}-b_{n}\right) t^{n \tau}, \quad b_{n}=\sum_{j=0}^{n} a_{j} a_{n-j} .
$$

If we apply the Laplace transform, we obtain

$$
\Lambda(\alpha, \beta, \gamma, \lambda) s^{\beta-\alpha \gamma}\left(s^{\alpha}-\lambda\right)^{\gamma} \sum_{n=0}^{\infty} a_{n} \frac{\Gamma(\xi n+1)}{s^{\tau} n+1}=\sum_{n=0}^{\infty}\left(a_{n}-b_{n}\right) \frac{\Gamma(\xi n+1)}{s^{\tau} \xi+1}
$$


or equivalently

$$
\Lambda(\alpha, \beta, \gamma, \lambda)\left(s^{\alpha}-\lambda\right)^{\gamma} \sum_{n=0}^{\infty} a_{n} \frac{\Gamma(\xi n+1)}{s^{\tau} n+1-\beta+\alpha \gamma}=\sum_{n=0}^{\infty}\left(a_{n}-b_{n}\right) \frac{\Gamma(\xi n+1)}{s^{\tau} n+1} .
$$

By using the binomial theorem,

$$
\Lambda(\alpha, \beta, \gamma, \lambda)\left(s^{\alpha}-\lambda\right)^{\gamma}=\sum_{n=0}^{\infty} \frac{\Gamma(\gamma+1)}{\Gamma(k+1) \Gamma(\gamma-n+1)} s^{\alpha n}(-\lambda)^{\gamma-n}=\sum_{n=0}^{\infty} c_{n} s^{\alpha n},
$$

where

$$
c_{n}=\frac{\Gamma(\gamma+1)}{\Gamma(n+1) \Gamma(\gamma-n+1)}(-\lambda)^{\gamma-n}
$$

and then

$$
\Lambda(\alpha, \beta, \gamma, \lambda) \sum_{n=0}^{\infty} \frac{c_{n}}{s^{-\alpha n}} \sum_{n=0}^{\infty} a_{n} \frac{\Gamma(\xi n+1)}{s^{\xi} n+1-\beta+\alpha \gamma}=\sum_{n=0}^{\infty}\left(a_{n}-b_{n}\right) \frac{\Gamma(\xi n+1)}{s^{\tilde{\xi} n+1}} .
$$

Thus, we have

$$
\begin{array}{rr}
\Lambda(\beta, \alpha, \gamma, 0) \mathbb{D}_{\beta, \alpha, 0}^{\gamma}={ }^{C} \mathbb{D}^{\alpha} & \text { (Liouville-Caputo), } \\
\Lambda(\beta, \alpha, 0, \lambda) \mathbb{D}_{\beta, \alpha, \lambda}^{0}={ }^{C} \mathbb{D}^{\alpha} & \text { (Liouville-Caputo), } \\
\Lambda(\alpha, 0,-1, \alpha /(\alpha-1)) \mathbb{D}_{\alpha, 0, \alpha /(\alpha-1)}^{-1}={ }^{\mathrm{AB}} \mathbb{D}^{\alpha} & \text { (Atangana-Baleanu), } \\
\Lambda(1,0,-1, \alpha /(\alpha-1)) \mathbb{D}_{\alpha, 0, \alpha /(\alpha-1)}^{-1}={ }^{C} \mathbb{D}^{\alpha} & \text { (Caputo-Fabrizio). }
\end{array}
$$

\subsection{Fractional Liouville-Caputo Logistic Differential Equation}

Let us consider the Liouville-Caputo fractional logistic differential equation

$$
\mathrm{C}_{\mathbb{D}^{\alpha}} x(t)=x(t)(1-x(t)),
$$

where ${ }^{C} \mathbb{D}^{\alpha}$ is defined in (10). Since

$$
\begin{aligned}
& \Lambda(\beta, \alpha, \gamma, 0) \mathbb{D}_{\beta, \alpha, 0}^{\gamma}={ }^{C} \mathbb{D}^{\alpha}, \\
& \Lambda(\beta, \alpha, 0, \lambda) \mathbb{D}_{\beta, \alpha, \lambda}^{0}={ }^{C} \mathbb{D}^{\alpha},
\end{aligned}
$$

by applying the Laplace transform, taking into account (19) and (18), we obtain

$$
\sum_{n=1}^{\infty} \frac{\Gamma(\xi n+1)}{s^{n \xi-\alpha+1}} a_{n}=\sum_{n=0}^{\infty}\left(a_{n}-b_{n}\right) \frac{\Gamma(n \xi+1)}{s^{n \xi+1}} .
$$

For $\xi=\alpha$, equating coefficients we have the following recurrence relation for the coefficients in the power series expansion (19)

$$
a_{1}=\frac{a_{0}-b_{0}}{\Gamma(1+\alpha)}, \quad a_{n}=\frac{\Gamma((n-1) \alpha+1)}{\Gamma(n \alpha+1)}\left(a_{n-1}-b_{n-1}\right) .
$$

We have included in Figures 2 and 3 some plots of the logistic function, solution to (1) with $x(0)=1 / 2$, as well as some approximations of the solution to the Liouville-Caputo fractional logistic differential Equation (20). 

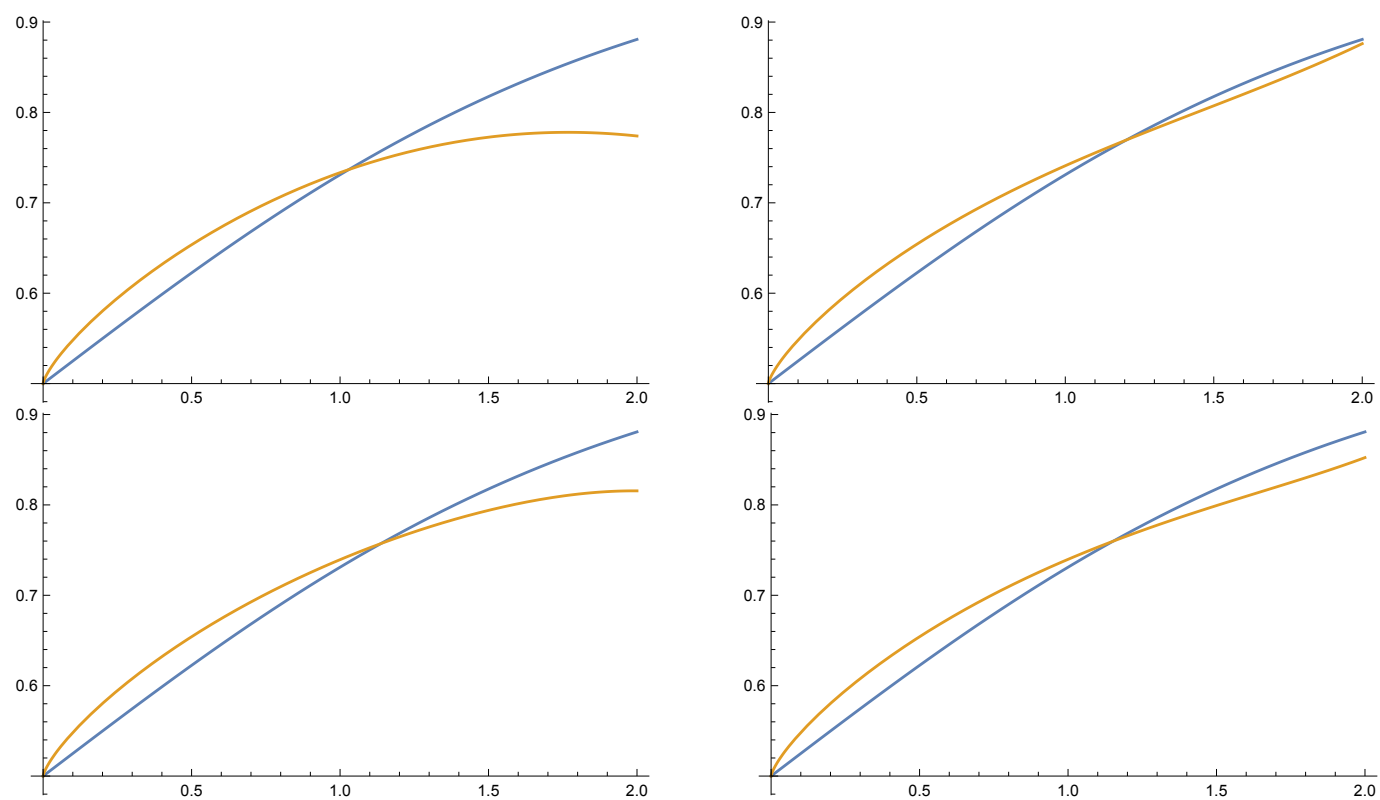

Figure 2. Logistic function solution to Equation (1) with $x(0)=1 / 2$, in blue, as well as some approximations of the solution to the Caputo fractional logistic differential Equation $(20)$ in $[0,2]$ for $\alpha=0.75$, in orange. From left to right and top to bottom the approximations are shown for $n=3$, $n=5, n=7$, and $n=9$. From these figures, one must use $\alpha$ closer to one as shown in Figure 3 in order to approximate the classical solution.
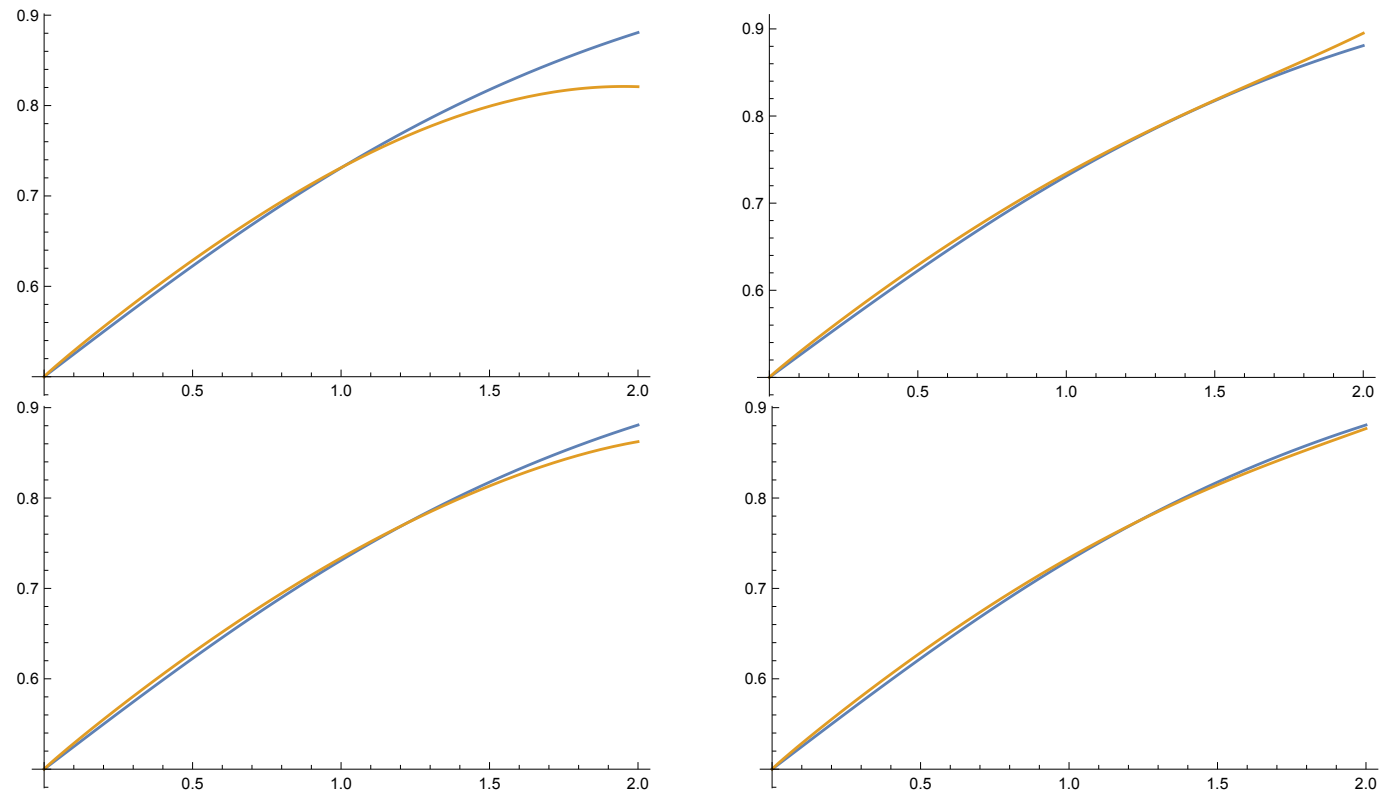

Figure 3. Logistic function solution to (1) with $x(0)=1 / 2$, in blue, as well as some approximations of the solution to the Caputo fractional logistic differential Equation (20) in $[0,2]$ for $\alpha=0.95$, in orange. From left to right and top to bottom the approximations are shown for $n=3, n=5, n=7$, and $n=9$.

\subsection{Atangana-Baleanu Logistic Differential Equation}

Let us consider

$$
{ }^{\mathrm{AB}} \mathbb{D}^{\alpha} x(t)=x(t)(1-x(t)),
$$

where ${ }^{A B} \mathbb{D}^{\alpha} f(t)$ is the Atangana-Baleanu derivative defined in (12).

Since

$$
\Lambda(\alpha, 0,-1, \alpha /(\alpha-1)) \mathbb{D}_{\alpha, 0, \alpha /(\alpha-1)}^{-1}={ }^{\mathrm{AB}} \mathbb{D}^{\alpha},
$$


if we apply the Laplace transform to (22), by using (19) and (18) we obtain

$$
\mathcal{L}\left[{ }^{\mathrm{AB}} \mathbb{D}^{\alpha} x(t)\right](s)=\sum_{n=0}^{\infty}\left(a_{n}-b_{n}\right) \frac{\Gamma(n \xi+1)}{s^{n \xi+1}} .
$$

Thus, for $\xi=\alpha$,

$$
\frac{B(\alpha)}{(1-\alpha)\left(s^{\alpha}+\frac{\alpha}{1-\alpha}\right)} \sum_{n=1}^{\infty} a_{n} \frac{\Gamma(n \alpha+1)}{s^{(n-1) \alpha+1}}=\sum_{n=0}^{\infty}\left(a_{n}-b_{n}\right) \frac{\Gamma(n \alpha+1)}{s^{n \alpha+1}} .
$$

Hence,

$$
\begin{aligned}
& \frac{B(\alpha)}{(1-\alpha)} \sum_{n=1}^{\infty} a_{n} \frac{\Gamma(n \alpha+1)}{s^{(n-1) \alpha+1}} \\
& =\sum_{n=0}^{\infty}\left(a_{n}-b_{n}\right) \frac{\Gamma(n \alpha+1)}{s^{\alpha(n-1)+1}}+\frac{\alpha}{1-\alpha} \sum_{n=0}^{\infty}\left(a_{n}-b_{n}\right) \frac{\Gamma(n \alpha+1)}{s^{n \alpha+1}} .
\end{aligned}
$$

Equating the coefficients, we obtain

$$
\begin{aligned}
& a_{1}=\frac{a_{0}-b_{0}+b_{1}(\alpha-1) \Gamma(\alpha)}{(B(\alpha)+\alpha-1) \Gamma(\alpha)}, \\
& a_{n}=\frac{(\alpha-1) b_{n}+\frac{\alpha\left(a_{n-1}-b_{n-1}\right) \Gamma((n-1) \alpha+1)}{\Gamma(n \alpha+1)}}{B(\alpha)+\alpha-1} .
\end{aligned}
$$

By using

$$
b_{n}=2 a_{0} a_{n}+\sum_{j=1}^{n-1} a_{j} a_{n-j}
$$

we finally obtain the initial step in terms of the initial condition

$$
a_{1}=\frac{\left(a_{0}-1\right) a_{0}}{\Gamma(\alpha)\left(\left(2 a_{0}-1\right)(\alpha-1)-B(\alpha)\right)},
$$

as well the recurrence relation for the coefficients

$$
a_{n}=\frac{(1-\alpha) \sum_{j=1}^{n-1} a_{j} a_{n-j}+\frac{\alpha \Gamma(\alpha(n-1)+1)\left(\sum_{j=1}^{n-1} a_{j} a_{n-j}+\left(2 a_{0}-1\right) a_{n-1}\right)}{\Gamma(\alpha n+1)}}{\left(2 a_{0}-1\right)(\alpha-1)-B(\alpha)},
$$

which in the limit as $\alpha \rightarrow 1$ converge to (21).

We have included in Figures 4 and 5 some plots of the logistic function, solution to (1) with $x(0)=1 / 2$, as well as some approximations of the solution to the Atangana-Baleanu logistic differential Equation (22). 

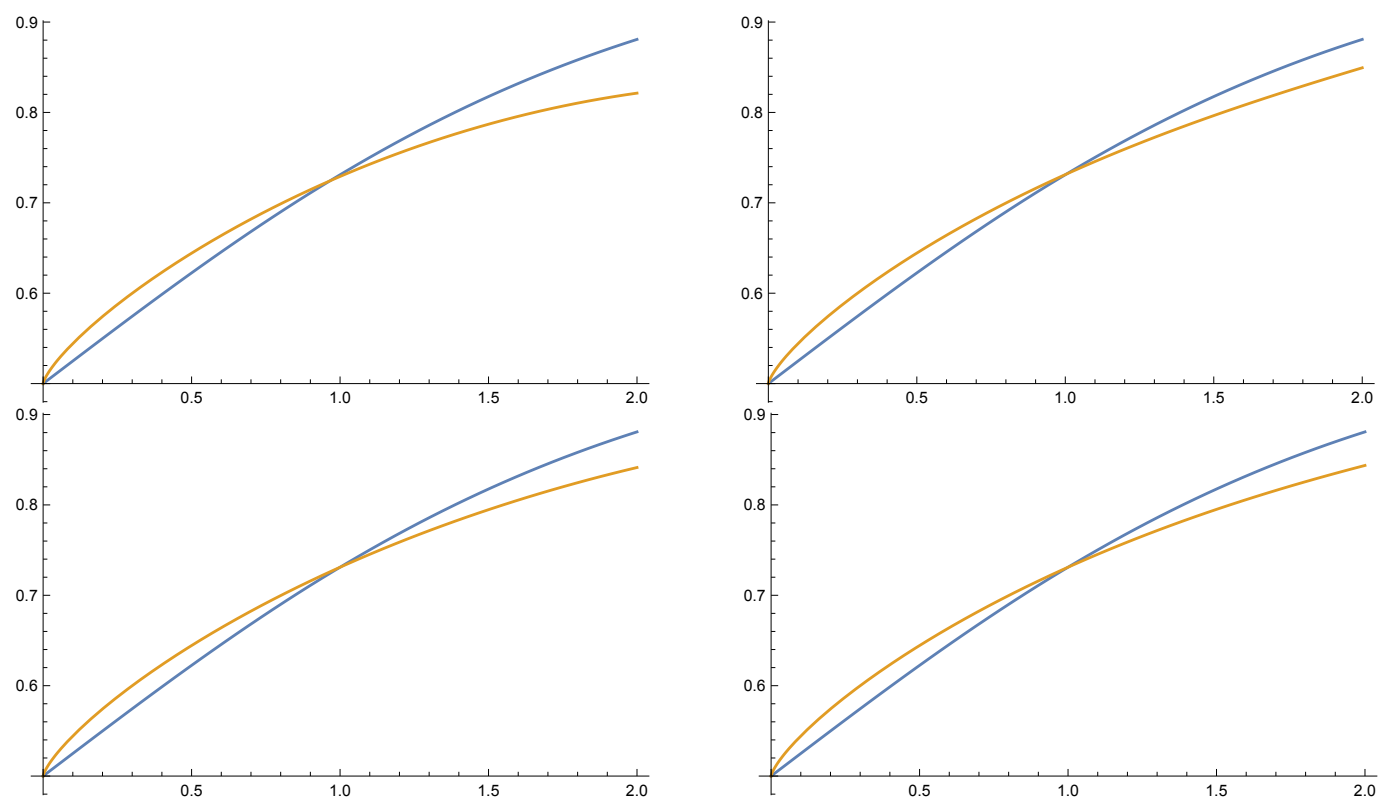

Figure 4. Logistic function solution to (1) with $x(0)=1 / 2$, in blue, as well as some approximations of the solution to the Atangana-Baleanu logistic differential equation in $[0,2]$ for $\alpha=0.75$, in orange. From left to right and top to bottom the approximations are shown for $n=3, n=5, n=7$, and $n=9$. From these figures, one must use $\alpha$ closer to one as shown in Figure 5 in order to approximate the classical solution.
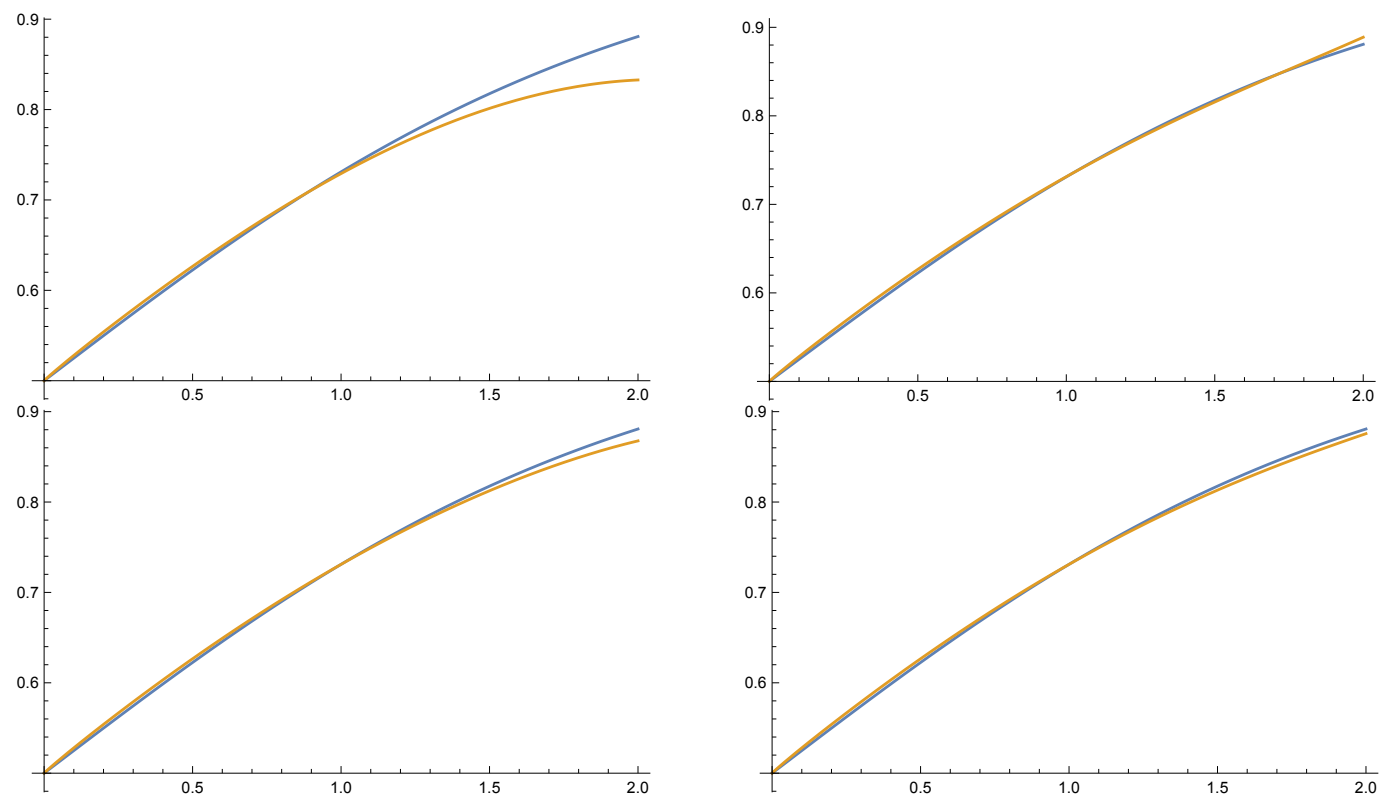

Figure 5. Logistic function solution to (1) with $x(0)=1 / 2$, in blue, as well as some approximations of the solution to the Atangana-Baleanu logistic differential equation in [0,2] for $\alpha=0.95$, in orange. From left to right and top to bottom the approximations are shown for $n=3, n=5, n=7$, and $n=9$.

\subsection{Caputo-Fabrizio Logistic Differential Equation}

Let us consider

$$
\mathrm{CF}^{\alpha} x(t)=x(t)(1-x(t)),
$$

where ${ }^{C F} \mathbb{D}^{\alpha}$ is the Caputo-Fabrizio derivative introduced in (14).

Since

$$
\Lambda(1,0,-1, \alpha /(\alpha-1)) \mathbb{D}_{\alpha, 0, \alpha /(\alpha-1)}^{-1}={ }^{C F} \mathbb{D}^{\alpha},
$$


if we apply the Laplace transform to (29), we obtain

$$
\sum_{n=1}^{\infty} \frac{\Gamma(n \xi+1)}{(\alpha(1-s)+s)} \frac{a_{n}}{s^{n \xi}}=\sum_{n=0}^{\infty}\left(a_{n}-b_{n}\right) \frac{\Gamma(n \xi+1)}{s^{n \xi+1}},
$$

which, if we fix $\xi=\alpha$, in the limit as $\alpha \rightarrow 1^{-}$, gives (5). Equivalently,

$$
\sum_{n=1}^{\infty} \Gamma(n \xi+1) \frac{a_{n}}{s^{n \xi}}=(\alpha+(1-\alpha) s) \sum_{n=0}^{\infty}\left(a_{n}-b_{n}\right) \frac{\Gamma(n \xi+1)}{s^{n \xi+1}},
$$

which can be rewritten as

$$
\sum_{n=1}^{\infty} \Gamma(n \xi+1) \frac{a_{n}}{s^{n \xi}}=\alpha \sum_{n=0}^{\infty}\left(a_{n}-b_{n}\right) \frac{\Gamma(n \xi+1)}{s^{n \xi+1}}+(1-\alpha) \sum_{n=0}^{\infty}\left(a_{n}-b_{n}\right) \frac{\Gamma(n \xi+1)}{s^{n \xi}} .
$$

Let $\xi=1$. If we equate the coefficients, we obtain

$$
a_{n}=\frac{1}{n}\left(a_{n-1}-b_{n-1}+n b_{n} \frac{\alpha-1}{\alpha}\right)
$$

which in the limit as $\alpha \rightarrow 1$ converges to (4). This relation can be obtained from [18] (Equation (8)).

We have included in Figures 6 and 7 some plots of the logistic function, solution to (1) with $x(0)=1 / 2$, as well as some approximations of the solution to the Caputo-Fabrizio logistic differential Equation (29).
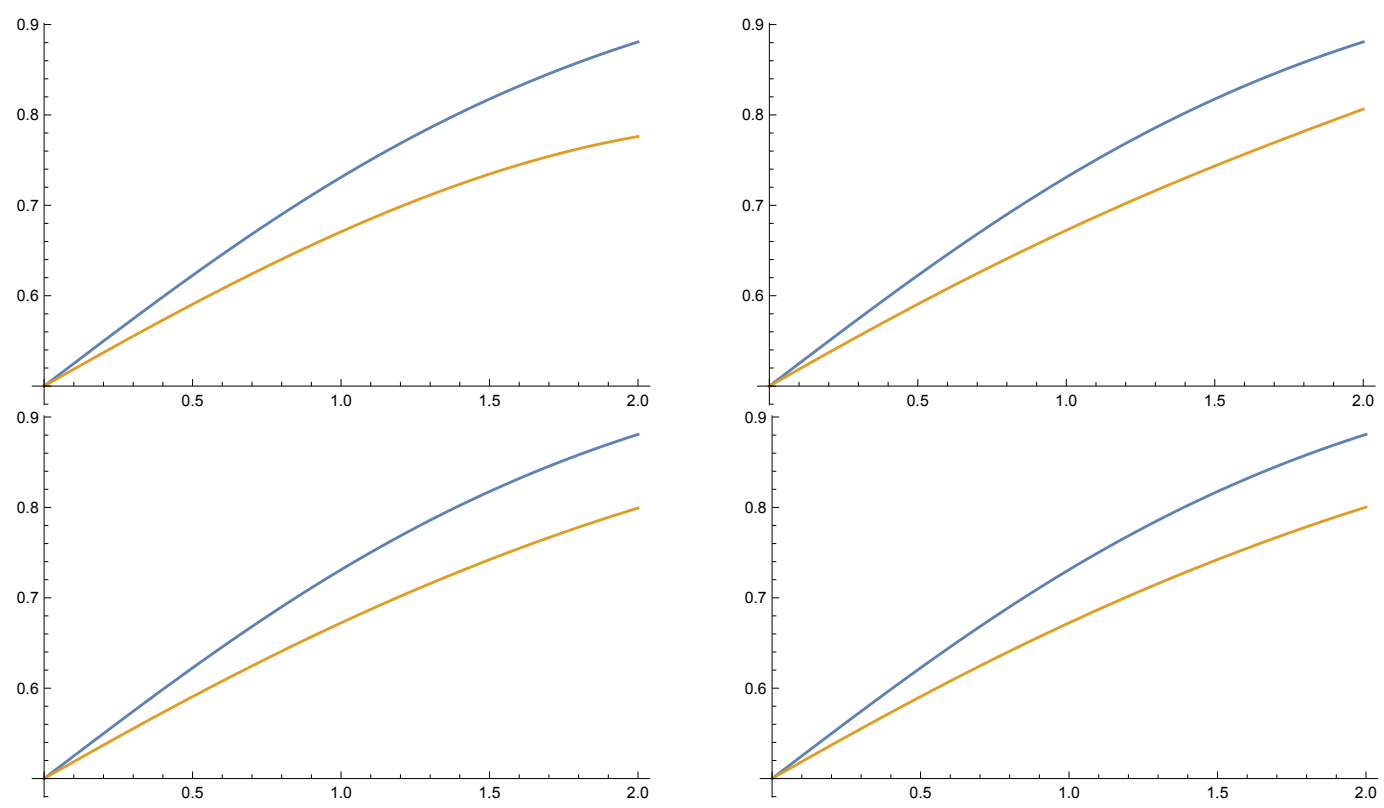

Figure 6. Logistic function solution to (1) with $x(0)=1 / 2$, in blue, as well as some approximations of the solution to the Caputo-Fabrizio logistic differential Equation (29) in $[0,2]$ for $\alpha=0.75$, in orange. From left to right and top to bottom the approximations are shown for $n=3, n=5, n=7$, and $n=9$. As in the previous cases, from these figures one must use $\alpha$ closer to one as shown in Figure 7 in order to approximate the classical solution. 

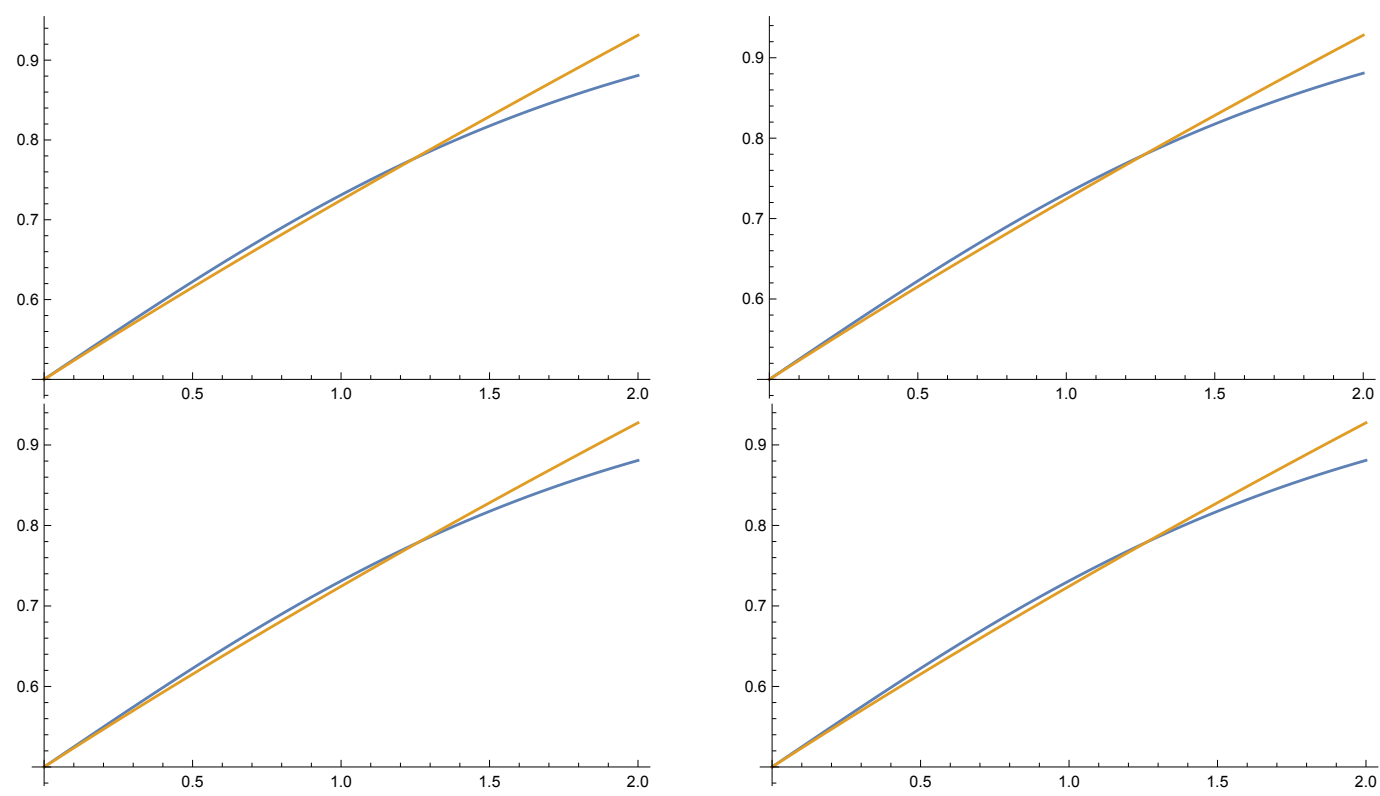

Figure 7. Logistic function solution to (1) with $x(0)=1 / 2$, in blue, as well as some approximations of the solution to the Caputo-Fabrizio logistic differential Equation (29) in $[0,2]$ for $\alpha=0.95$, in orange. From left to right and top to bottom the approximations are shown for $n=3, n=5, n=7$, and $n=9$.

Moreover, in Figure 8, we show a comparison between the results in [18] in implicit form, and the results presented here in terms of recurrence relation for the coefficients in the power series expansion. For this last comparison, we have chosen $\alpha=0.9$.
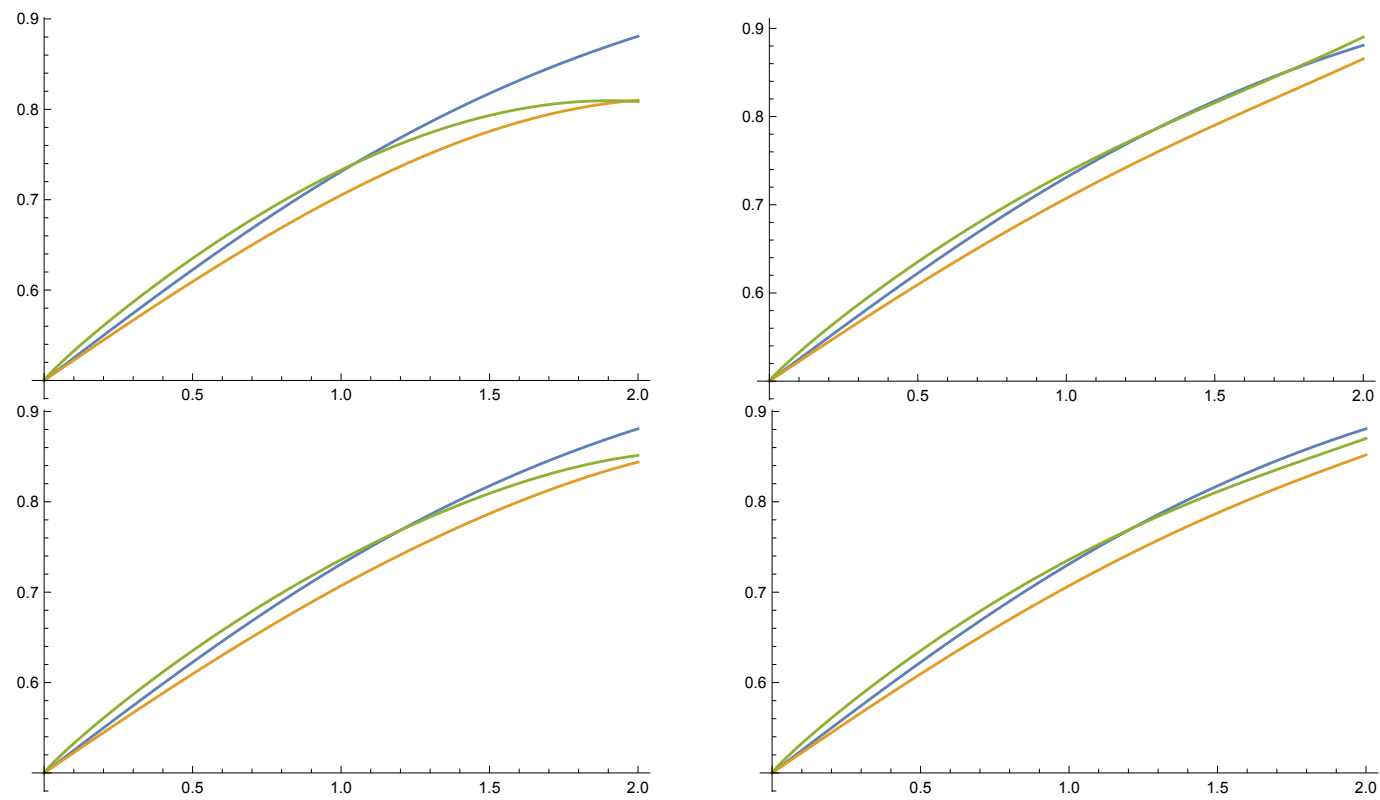

Figure 8. In [0,2] for $\alpha=0.9$, logistic function solution to (1) with $x(0)=1 / 2$, in blue, some approximations of the solution to the Caputo-Fabrizio logistic differential Equation (29), as well as the solution given in [18] in orange. From left to right and top to bottom the approximations are shown for $n=3, n=5, n=7$, and $n=9$.

\section{Conclusions}

The Prabhakar fractional calculus, based on the three-parameter generalization of the Mittag-Leffler function, provides some physical examples such as anomalous phenomena showing the need for an extension of ordinary calculus based on the Prabhakar function [15]. 
We contribute to the study of the fractional logistic differential equation in the setting of Prabhakar fractional calculus by solving that logistic equation in some cases.

Author Contributions: All authors contributed equally to this work. All authors have read and agreed to the published version of the manuscript.

Funding: This work has been partially supported by the Agencia Estatal de Investigación (AEI) of Spain under Grant PID2020-113275GB-I00, cofinanced by the European Community fund FEDER, and Xunta de Galicia, grant ED431C 2019/02 for Competitive Reference Research Groups (2019-2022).

Institutional Review Board Statement: Not applicable.

Informed Consent Statement: Not applicable.

Data Availability Statement: Not applicable.

Acknowledgments: The authors thank the three anonymous referees and editor for their valuable comments which improved a preliminary version of this material.

Conflicts of Interest: The authors declare no conflict of interest.

\section{References}

1. Area, I.; Nieto, J.J. Power series solution of the fractional logistic equation. Phys. A Stat. Mech. Its Appl. 2021, 573, 125947. [CrossRef]

2. Wolfram Research, Inc. Mathematica, Version 12.1; Wolfram Research, Inc.: Champaign, IL, USA, 2020.

3. Thieme, H.R. Mathematics in Population Biology, Princeton series in Theoretical and Computational Biology; Princeton University Press: Princeton, NJ, USA, 2003.

4. Saito, T. A Logistic Curve in the SIR Model and Its Application to Deaths by COVID-19 in Japan. MedRxiv 2020. [CrossRef]

5. Pelinovsky, E.; Kurkin, A.; Kurkina, O.; Kokoulina, M.; Epifanova, A. Logistic equation and COVID-19. Chaos Solitons Fractals 2020, 140, 110241. [CrossRef] [PubMed]

6. Ortigueira, M.; Bengochea, G. A new look at the fractionalization of the logistic equation. Phys. A Stat. Mech. Its Appl. 2017, 467, 554-561. [CrossRef]

7. Area, I.; Losada, J.; Nieto, J.J. A note on the fractional logistic equation. Physica A 2016, 444, 182-187. [CrossRef]

8. D'Ovidio, M.; Loreti, P.; Sarv Ahrabi, S. Modified fractional logistic equation, Phys. A Stat. Mech. Its Appl. 2018, 505, 818-824. [CrossRef]

9. El-Sayed, A.M.A.; El-Mesiry, A.E.M.; El-Saka, H.A.A. On the fractional-order logistic equations. Appl. Math. Lett. 2007, 20, 817-823. [CrossRef]

10. Kaharuddin, L.N.; Phang, C.; Jamaian, S.S. Solution to the fractional logistic equation by modified Eulerian numbers. Eur. Phys. J. Plus 2020, 135, 229. [CrossRef]

11. West, B.J. Exact solution to fractional logistic equation. Physica A 2015, 429, 103-108. [CrossRef]

12. Izadi, M.; Srivastava, H.M. A discretization approach for the nonlinear fractional logistic equation. Entropy 2020, 22, 1328. [CrossRef] [PubMed]

13. Izadi, M.; Srivastava, H.M. Numerical approximations to the nonlinear fractional-order logistic population model with fractionalorder Bessel and Legendre bases. Chaos Solitons Fractals 2021, 145, 110779. [CrossRef]

14. Oldham, K.B.; Spanier, J. The Fractional Calculus: Theory and Applications of Differentiation and Integration to Arbitrary Order; Academic Press: Cambridge, MA, USA, 1974.

15. Giusti, A.; Colombaro, I.; Garra, R.; Garrappa, R.; Polito, F.; Popolizio, M.; Mainardi, F. A practical guide to Prabhakar fractional calculus. Frac. Calcul. Appl. Anal. 2020, 23, 9-54. [CrossRef]

16. Prabhakar, T.R. A singular integral equation with a generalized Mittag- Leffler function in the kernel. Yokohama Math. J. 1971, 19, 7-15.

17. Samko, S.; Kilbas, A.A.; Marichev, O. Fractional Integrals and Derivatives; Taylor \& Francis: Abingdon, UK, 1993.

18. Nieto, J. Solution of a fractional logistic ordinary differential equation. Appl. Math. Lett. 2022, 123, 107568. [CrossRef]

19. Srivastava, H.M. An introductory overview of fractional-calculus operators based upon the Fox-Wright and related higher transcendental functions. J. Adv. Eng. Comput. 2021, 5, 135-166.

20. Abramowitz, M.; Stegun, I.A. Handbook of Mathematical Functions, 9th ed.; Dover: New York, NY, USA, 1972.

21. Gorenflo, R.; Kilbas, A.A.; Mainardi, F.; Rogosin, S. Mittag-Leffler functions. In Theory and Applications; Springer Monographs in Mathematics; Springer: Berlin, Germany, 2014.

22. Garra, R.; Garrappa, R. The Prabhakar or three parameter Mittag-Leffler function: Theory and application. Commun. Nonlin. Sci. Numer. Simul. 2018, 56, 314-329. [CrossRef]

23. Caputo, M. Linear model of dissipation whose $Q$ is almost frequency independent. II. Geophys. J. Int. 1967, 13, 529-539. [CrossRef]

24. Atangana, A.; Baleanu, D. New fractional derivatives with non-local and non-singular kernel. Theory and application to heat transfer model. Ther. Sci. 2016, 20, 763-769. [CrossRef] 
25. Caputo, M.; Fabrizio, M. On the singular kernels for fractional derivatives. Some applications to partial differential equations. Progr. Fract. Differ. Appl. 2021, 7, 1-21.

26. Losada, J.; Nieto, J.J. Fractional integral associated to fractional derivatives with nonsingular kernels. Progr. Fract. Differ. Appl. 2021, 7, 79-82. 